\title{
Coleópteros (Bostrichidae, Platypodidae e Scolytidae) em um fragmento de cerrado da baixada Cuiabana ${ }^{1}$
}

\author{
Coleopterans (Bostrichidae, Platypodidae e Scolytidae) in fragment of \\ savannah in baixada Cuiabana
}

\author{
José Renato Maurício da Rocha ${ }^{2(*)}$ \\ Alberto Dorval ${ }^{3}$ \\ Otávio Peres Filho ${ }^{4}$ \\ Alex Lima da Silva ${ }^{2}$
}

\section{Resumo}

O objetivo desta pesquisa foi qualificar e quantificar as populações de Bostrichidae, Platypodidae e Scolytidae que ocorrem associadas a um fragmento de cerrado nos períodos de seca (maio a outubro) e de chuva (novembro a abril), no município de Cuiabá, estado de Mato Grosso. As coletas foram quinzenais e ocorreram de abril de 2008 a março de 2009. Foram utilizadas seis armadilhas etanólicas, iscadas com álcool etílico combustível hidratado. Os exemplares coletados foram triados, contados e identificados nos Laboratórios de Proteção Florestal da Universidade Federal de Mato Grosso e da Universidade Federal do Paraná. Foram realizados estudos faunísticos de dominância, constância, frequência, abundância e de diversidade. Também foi desenvolvido estudo de flutuação populacional, de correlação com a tempertura, umidade relativa, precipitação pluvial e testes de médias entre as espécies. Foram identificadas 25 espécies, sendo cinco de Bostrichidae, duas de Platypodidae e 18 de Scolytidae. Foi coletado um total de 874 indivíduos, dos quais 588 foram coletados no período de seca e 286 no período de chuva. Bostrichopsis uncinata, Cryptocarenus diadematus, Xyleborus affinis e Xyleborus retusus foram as espécies mais numerosas no período de seca, enquanto Bostrichopsis uncinata, Xyleborus affinis e Xyleborus ferrugineus foram

I Trabalho de parte da dissertação de mestrado, do Programa de Pós-Graduação em Ciências Florestais e Ambientais, da Faculdade de Engenharia Florestal, Universidade Federal de Mato Grosso.

2 Mestrando do Programa de Pós-Graduação em Ciências Florestais eAmbientais, da Faculdade de Engenharia Florestal/UFMT. Endereço:Av. Fernando Corrêa da Costa s/n - Coxipó da Ponte. Cuiabá/MT, CEP: 79.060900, E-mail:jrm_rocha@hotmail.com; alexperplex@hotmail.com ${ }^{*}$ autor para correspondência

3 Dr.; Engenheiro Florestal; Professor da Faculdade de Engenharia Florestal e do Programa de Pós-Graduação em Ciências Florestais e Ambientais/Universidade Federal do Mato Grosso. Endereço:Av. Fernando Corrêa da Costa s/n - Coxipo da Ponte.Cuiabá, MT, CEP:78.060-900,E-mail:a.dorval@hotmail.com; peres@ufmt.br

4 Dr.; Engenheiro Florestal; Professor da Faculdade de Engenharia Florestal e coordenador do Programa de Pós-Graduação em Ciências Florestais e Ambientais/Universidade Federal do Mato Grosso. Endereço: Fernando Corrêa da Costa s/n - Coxipo da Ponte.Cuiabá,MT,CEP:78.060-900,E-mail:a.dorval@hotmail.com.

Recebido para publicação em 19/08/2010 e aceito em I2/04/201।

Ambiência Guarapuava (PR) v.7 n.I p.89 - 101 Jan./Abr. 201 I ISSN 1808 - 025I

DOI: $10.5777 /$ ambiencia.2011.01.07 
mais representativas no período de chuvas. As espécies Cryptocarenus diadematus, Xyleborus affinis e Xyleborus retusus ocorreram como dominante, muito abundante, muito frequente e constante somente no período seco, enquanto que no período de chuvas Xyleborus affinis ocorreu como super dominante, super abundante, super frequente e constante. No período de seca, os índices de Equitatibilidade e de Shannon-Wiener foram mais expressivos quando comparados com os obtidos nos meses de chuva.

Palavras-chave: Coleobrocas; Besouros-de-ambrosia; armadilha etanólica.

\section{Abstract}

The purpose of this research was to qualify and quantify the populations of Bostrichidae, Platypodidae and Scolytidae that occur associated with a piece of Brazilian savanna during the dry season (May to October) and the wet season (November to April) in Cuiabá city, Mato Grosso. The collections occurred every 15 days from April 2008 to March 2009. Six ethanolic traps were used, and baited with hydrated ethanol fuel. The collected samples were sorted, counted and identified at the Forest Protection Laboratory of the Federal University of Mato Grosso and the Federal University of Paraná. There were many tests conducted, faunal dominance, constancy, frequency, abundance and diversity studies. There were also developed population fluctuation studies, correlation with temperature, relative humidity, rainfall and mean tests between collected species and weather periods analyzed. We identified 25 species, five of Bostrichidae, two Platypodidae and 18 Scolytidae. We collected a total of 874 specimens, 588 were collected during the dry period and 286 during the wet season. Bostrichopsis uncinata, Cryptocarenus diadematus, Xyleborus affinis, and Xyleborus retusus were the most common species in the dry season, whilst in the wet season Bostrichopsis uncinata, Xyleborus affinis and Xyleborus ferrugineus had a higher representation. The species Cryptocarenus diadematus, Xyleborus affinis and Xyleborus retusus occurred as the dominant, very abundant, very frequent, yet only constant during the dry season, whereas during the rainy season Xyleborus affinis occurred clearly to be the extremely dominant, extremely abundant, extremely frequent and constant. During the dry season, the indexes of Equitability and Shannon-Wiener were more significant when compared with wet season.

Key words: Woodborers; Ambrosia beetles; Ethanolic trap.

\section{Introdução}

A vegetação de cerrado abriga uma entomofauna adaptada a condições ecológicas diferenciadas, como período de seca prolongada, ocorrência de fogo, alta temperatura e baixa umidade relativa. Uma variedade de espécies do grupo considerado brocas de madeira ocorre neste ambiente, associadas a uma diversidade de espécies 
de vegetais. São responsáveis pela primeira fase de ciclagem de nutrientes presentes na matéria orgânica das partes lenhosas da vegetação (DORVAL, 2002).

Neste ambiente, pode-se capturar uma grande quantidade de espécimens das familias Bostrichidae, Platypodidae e Scolytidae (DORVAL e PERES-FILHO, 2001). As espécies-brocas da família Bostrichidae são conhecidas como besouros pulverizadores, pois, ao abrirem as galerias, transformam a madeira em pó, depreciando o valor de produtos, como peças estruturais e lâminas (HILL et al. 2003; MATOSKI, 2005). São considerados de difícil controle pois, como todas as coleobrocas, passam a maior parte de sua vida no interior do hospedeiro (BERTIFILHO,1997).

Da família Bostrichidae são conhecidas muitas espécies tidas como pragas florestais tais como Apates terebrans Pallas, 1772, Dinoderus minutus (Fabricius, 1775) (SILVA et al.,1968), Micrapate brasiliensis Lene,1898, Rhizopertha dominica (Fabicius, 1775), Xilopethella picea (Olivier, 1790), Xyloprista exacantha (Faimaire,1892) e Bostrichopsis uncinata (Germar, 1824) (DORVAL, 2002).

Nas familias Scolytidae e Platypodidae encontram-se espécies conhecidas como besouros-de-ambrosia, responsáveis pela abertura de galerias e manchamento da madeira. As coleobrocas destas famílias perfuram galerias de nidificação em hospedeiros estressados, doentes e em madeiras recém-cortadas (MARINONI et al. 2001). Isto ocorre devido à liberação de voláteis químicos oriundos da fermentação da madeira e que são atrativos para estas espécies (SIMEONE, 1965; FURNISS e CAROLIN, 1977).

Este trabalho foi realizado com a intenção de contribuir para o conhecimento da diversidade e flutuação populacional das espécies de bostriquídeos, platipodídeos e escolitídeos em um fragmento de cerrado durante os meses de seca e de chuvas na zona rural no município de Cuiabá, estado de Mato Grosso.

\section{Material e Métodos}

O estudo foi realizado de abril de 2008 a março de 2009 na fazenda Mutuca, localizada no município de Cuiabá-MT $\left(15^{\circ} 18^{\prime} \mathrm{S}\right.$ e $\left.55^{\circ} 59^{\prime} \mathrm{W}\right)$, em um fragmento de 3,56 ha com vegetação de cerrado remanescente e não alterado, pertencente à área de reserva legal da propriedade.

O relevo da área é plano a suavemente ondulado, com a altitude média de 800 metros acima do nível do mar. O solo da região é do tipo areia quartzosa álica, de textura arenosa, destituído de minerais primários, pouco resistentes ao intemperismo e de baixa fertilidade. A composição da vegetação natural da região é do tipo cerrado ralo, campos, matas ciliares e cerradões (CONCEIÇÃO, 1997). O clima da região é classificado como "Aw" (Köppen), com temperatura média anual de $26^{\circ} \mathrm{C}$ e precipitação pluviométrica média anual de $1400 \mathrm{~mm}$.

Neste levantamento foram utilizadas seis armadilhas etanólicas modelo escolitídeo/ Curitiba modificada (MARQUES, 1984), dispostas em dois transectos contendo três armadilhas por transecto com uma distância de $30 \mathrm{~m}$ entre si e entre transecto. As armadilhas foram instaladas a $1,5 \mathrm{~m}$ da superfície do solo em relação à borda do funil coletor. Após cada coleta, as armadilhas foram abastecidas com etanol combustível $93 \%$ como atrativo e com etanol $70 \%$ no frasco coletor. 
As coletas ocorreram quinzenalmente nos períodos de seca (maio a outubro) e de chuva (novembro a abril) e os espécimens coletados foram acondicionados em recipientes plásticos, identificados com o número da armadilha e data de coleta. Foram então transportados para o Laboratório de Proteção Florestal da Universidade Federal de Mato Grosso para triagem e posterior secagem em estufa a $60^{\circ} \mathrm{C}$ por 72 horas. Após esta fase, as amostras de cada armadilha foram acondicionadas em recipientes plasticos de 10 $\mathrm{ml}$ devidamente vedados e identificados.

Os espécimens capturados foram montados e identificados taxonomicamente por comparação com material entomológico depositado nas coleções dos Laboratórios de Proteção Florestal, das Faculdades de Engenharia Florestal, da Universidade Federal de Mato Grosso e Universidade Federal do Paraná.Também foram enviados exemplares aos especialistas Prof.Dr. Eli Nunes Marques (Scolytidae) da Universidade Federal do Paraná, e Dr. Edson Possidônio Teixeira (Bostrichidae) do Instituto Agronomico de Campinas, para a identificação taxonômica de alguns exemplares.

A análise qualitativa e quantitativa para os períodos de seca (maio a outubro) e de chuva (novembro a abril) foram feitas mediante contagem direta dos indivíduos, e os cálculos da frequencia, constância, dominância, abundância e diversidade, para cada período, foram feitos através do software ANAFAU (MORAES e SILVEIRA NETO, 2003).

Também foram realizados estudos de flutuação populacional e de correlação de Pearson ao nível de significância de 1\% e 5\% de probabilidade entre temperatura, umidade relativa, precipitação pluvial e as espécies mais representadas, ou seja, as que ocorreram na análise faunística anual como dominantes, muito abundantes, muito frequentes e constantes. Para efeito de discussão foram consideradas somente as correlações com valor de r acima de $70 \%(0,7)$, por considerar que possa explicar melhor a correlação com os fatores envolvidos. Para esta análise foi utilizado o software SAEG 5.0, com o auxílio do Dr Joadil Gonçalves Abreu, da Universidade Federal de Mato Grosso.

Os valores originais das coletas foram transformados pela equação $\log$ $(\mathrm{x}+1)$ para efeito de cálculos estatísticos $\mathrm{e}$ também foram submetidos aos testes de normalidade de Lilliefors (CAMPOS, 1976) e de homogeneidade de variância de Cochran (COSTA NETO, 1977). Foi utilizado o delineamento inteiramente casualizado em esquema fatorial $25 \times 2$ ( 25 espécies por dois períodos (seca e chuva) com seis armadilhas/ repetições, constituindo 300 parcelas).

As médias das coletas de cada espécie nos perídos de seca e de chuva foram comparadas entre si pelo teste de Scott-Knot a $5 \%$ de probabilidade.

\section{Resultados e Discussão}

Neste ambiente, foram coletados 874 indivíduos, sendo que $67,27 \%$ do total foi capturado no período de seca e $32,73 \%$ no período de chuva. Resultado semelhante foi obtido por DORVAL (2002) que em vegetação de cerrado, no município de Cuiabá, coletou um total de 2.111 indivíduos, dos quais $61,34 \%$ foram coletados nos meses de seca. Esse autor atribuiu este resultado ao período de estiagem na região, que ocasiona queda de pequenos ramos e galhos, hospedeiros preferenciais para espécies de escolitídeos xilófagos, mielófagos e xilomicetófagos (MARINONI et al.2001). 
Foram identificadas 25 espécies, das quais cinco foram da família Bostrichidae, duas de Platypodidae e 18 de Scolytidae. No período seco ocorreram 22 espécies, tendo sido Scolytidae a família mais representativa com $72,73 \%$ das espécies identificadas e $56,97 \%$ dos indivíduos coletados. No período de chuva, ocorreram 20 espécies, sendo que novamente a família Scolytidae foi a mais representativa com $75 \%$ das espécies identificadas e 89,51\% dos indivíduos coletados (Tabela 1). Com resultados muito parecidos, Dorval (2002) constatou neste mesmo ambiente as familias Scolytidae e Bostrichidae como as mais representativas em termos de diversidade e Scolytidae a familia da qual se coletou a maior quantidade dos espécimens. No entanto, Carrano Moreira e PedrosaMacedo (1994) coletaram 818 escolitídeos distribuídos em 37 espécies, e atribuíram a baixa quantidade de indivíduos coletados à escassez na quantidade e qualidade dos resíduos florestais, que se constituem na principal fonte de alimento de várias espécies de escolitídeos.

Dezessete espécies foram comuns aos períodos de seca e de chuva. No entanto, as espécies Micrapate sp., Xyloperthela picea, e Xyleborus spinosulus ocorreram somente no período de seca, enquanto Bostrychopsis lamnifer, ocorreu somente no período de chuva (Tabela 2). Das espécies Coccotripes sp., Corthylus convexicauda, Xyleborus spinulosus, e Tricolus sp. foi coletado apenas um indivíduo, não sendo possível determinar qualquer tendência sobre as mesmas. Dorval (2002), em estudo de diversidade de coleópteros em vegetação de cerrado, observou a ocorrência de 19 espécies comuns aos períodos de seca e de chuva, alegando que a ocorrência de chuvas afeta drásticamente as condições ambientais, influenciando na seleção, colonização, desenvolvimento e disponibilidade de alimento para algumas espécies, e que, portanto, algumas espécies são encontradas em somente um dos períodos meteorológicos.

\section{Índices Faunísticos}

Sete espécies foram dominantes, quatro muito abundantes, cinco muito frequentes e doze constantes, considerando o período anual. No período seco, sete foram dominantes, quatro muito abundantes, quatro muito frequentes e dessezete constantes, enquanto nos meses de chuva, quatro ocorreram como dominantes duas muito abundantes e muito frequentes e dez constantes. Apenas B. uncinata ocorreu como dominante, muito abundante, muito

Tabela I. Riqueza de espécie e número de indivíduos coletados no período de seca e de chuva em um fragmento de cerrado no município de Cuiabá, estado de Mato Grosso, de abril/2008 a março/2009

\begin{tabular}{lllllllllll}
\hline \multirow{2}{*}{ Famílias } & \multicolumn{4}{c}{ Período de Seca } & \multicolumn{4}{c}{ Período de Chuva } \\
\cline { 2 - 11 } & \multicolumn{1}{c}{$\%$} & \multicolumn{1}{c}{$\%$} & $\mathrm{~N}$ & $\%$ & $\mathrm{M}$ & $\mathrm{R}$ & $\%$ & $\mathrm{~N}$ & $\%$ & $\mathrm{M}$ \\
\hline Bostrichidae & 4 & 18,18 & 218 & 37,07 & 54,50 & 3 & 15,00 & 25 & 8,74 & 8,33 \\
Platypodidae & 2 & 9,09 & 35 & 5,95 & 17,50 & 2 & 10,00 & 5 & 1,75 & 2,50 \\
Scolytidae & 16 & 72,73 & 335 & 56,97 & 20,94 & 15 & 75,00 & 256 & 89,51 & 17,07 \\
\hline Total & 22 & 100,00 & 588 & 100,00 & 26,73 & 20 & 100,00 & 286 & 100,00 & 14,30 \\
\hline
\end{tabular}

Nota: R: Riqueza de Espécies. N: Número de Indivíduos. $M=N / R$ 
Tabela 2. Família, gênero, espécie e quantidade de indivíduos coletados nos períodos de seca e chuva em um fragmento de cerrado, no município de Cuiabá, estado de Mato Grosso, de abril/2008 a março/2009

\begin{tabular}{llccc}
\hline \multirow{2}{*}{ Espécie } & \multirow{2}{*}{ Familia } & \multicolumn{3}{c}{ Indivíduos } \\
\cline { 3 - 5 } & & Seca & Chuva & Anual \\
\hline Bostrychopsis laminifer & Bostrichidae & - & 5 & 5 \\
Bostrychopsis uncinata & Bostrichidae & 144 & 18 & 162 \\
Micrapate sp. & Bostrichidae & 41 & - & 41 \\
Xyloperthela picea & Bostrichidae & 24 & - & 24 \\
Xyloprista sp. & Bostrichidae & 9 & 2 & 11 \\
Platypus linearis & Platypodidae & 34 & 4 & 38 \\
Platypus sp. & Platypodidae & 1 & 1 & 2 \\
Coccotrypes sp. & Scolytidae & - & 1 & 1 \\
Corthylus convexicauda & Scolytidae & 1 & - & 1 \\
Cryptocarenus diadematus & Scolytidae & 78 & 10 & 88 \\
Cryptocarenus heveae & Scolytidae & 3 & 4 & 7 \\
Cryptocarenus seriatus & Scolytidae & 11 & 3 & 14 \\
Cryptocarenus sp. & Scolytidae & 1 & 1 & 2 \\
Hypothenemus bolivianus & Scolytidae & 2 & 1 & 3 \\
Hypothenemus eruditus & Scolytidae & 36 & 6 & 42 \\
Hypothenemus obscurus & Scolytidae & 5 & 3 & 8 \\
Premnobius cavipennis & Scolytidae & 9 & 10 & 19 \\
Sampsonius dampfi & Scolytidae & 7 & 5 & 12 \\
Xyleborus affinis & Scolytidae & 86 & 163 & 249 \\
Xyleborus compactus & Scolytidae & 3 & 1 & 4 \\
Xyleborus ferrugineus & Scolytidae & 21 & 45 & 66 \\
Xyleborus retusus & Scolytidae & 62 & 2 & 64 \\
Xyleborus spinosulus & Scolytidae & 1 & - & 1 \\
Xyleborus spinulosus & Scolytidae & 9 & - & 9 \\
Tricolus sp. & Scolytidae & - & 1 & 1 \\
\hline Total & & 588 & 286 & 874 \\
\hline & & & &
\end{tabular}

frequente e constante, independentemente do período analisado. As espécies $C$. diadematus, $X$. affinis e $X$. retusus ocorreram como dominante, muito abundante, muito frequente e constante somente no período seco, enquanto que no período de chuvas $X$. affinis ocorreu como super dominante, super abundante, super frequente e constante (Tabela 3).
Resultado semelhante foi obtido por Dorval (2002), em vegetação de cerrado, para as espécies C. diadematus, C. heveae, C. seriatus, $H$. obscurus e $H$. eruditus que ocorreram nos períodos de seca e de chuva como dominantes, muito abundantes, constantes e com altas frequências de coletas. Flechtmann e Ottati (1996) também observaram, em vegetação de cerrado, a 
Tabela 3. Índices faunísticos para as espécies identificadas em um fragmento de cerrado no município de Cuiabá, estado de Mato Grosso, de abril/2008 a março/2009

\begin{tabular}{|c|c|c|c|c|c|c|c|c|c|c|c|c|}
\hline \multirow{2}{*}{ Espécies } & \multicolumn{4}{|c|}{ Seca } & \multicolumn{4}{|c|}{ Chuva } & \multicolumn{4}{|c|}{ Anual } \\
\hline & D & $A$ & $\mathrm{~F}$ & C & D & $A$ & $\mathrm{~F}$ & C & D & $A$ & $\mathrm{~F}$ & C \\
\hline Bostrychopsis laminifer & - & - & - & - & nd & c & $\mathrm{f}$ & $\mathrm{w}$ & nd & $\mathrm{r}$ & $\mathrm{pf}$ & $\mathrm{y}$ \\
\hline Bostrychopsis uncinata & $\mathrm{d}$ & $\mathrm{ma}$ & $\mathrm{mf}$ & $\mathrm{w}$ & $\mathrm{d}$ & $\mathrm{ma}$ & $\mathrm{mf}$ & $\mathrm{w}$ & $\mathrm{d}$ & $\mathrm{ma}$ & $\mathrm{mf}$ & $\mathrm{w}$ \\
\hline Micrapate sp. & $\mathrm{d}$ & $\mathrm{c}$ & $\mathrm{f}$ & $\mathrm{w}$ & - & - & - & - & $\mathrm{d}$ & $\mathrm{c}$ & $\mathrm{f}$ & $\mathrm{y}$ \\
\hline Xyloperthela picea & nd & c & $\mathrm{f}$ & $\mathrm{w}$ & - & - & - & - & nd & c & $\mathrm{f}$ & $\mathrm{w}$ \\
\hline Xyloprista sp. & nd & d & $\mathrm{pf}$ & $\mathrm{w}$ & nd & c & $\mathrm{f}$ & $\mathrm{y}$ & nd & $\mathrm{d}$ & $\mathrm{pf}$ & $\mathrm{w}$ \\
\hline Platypus linearis & $\mathrm{a}$ & c & $\mathrm{f}$ & $\mathrm{w}$ & nd & c & $\mathrm{f}$ & $\mathrm{w}$ & d & c & $\mathrm{f}$ & $\mathrm{w}$ \\
\hline Platypus sp. & nd & $\mathrm{r}$ & $\mathrm{pf}$ & $\mathrm{z}$ & nd & d & $\mathrm{pf}$ & $\mathrm{z}$ & nd & $\mathrm{r}$ & $\mathrm{pf}$ & z \\
\hline Coccotrypes sp. & - & - & - & - & nd & $\mathrm{d}$ & $\mathrm{pf}$ & $\mathrm{z}$ & nd & $\mathrm{r}$ & $\mathrm{pf}$ & z \\
\hline Corthylus convexicauda & nd & $\mathrm{r}$ & $\mathrm{pf}$ & $\mathrm{z}$ & - & - & - & - & nd & $\mathrm{r}$ & $\mathrm{pf}$ & z \\
\hline Cryptocarenus diadematus & $\mathrm{d}$ & $\mathrm{ma}$ & $\mathrm{mf}$ & $\mathrm{w}$ & $\mathrm{d}$ & c & $\mathrm{f}$ & $\mathrm{w}$ & $\mathrm{d}$ & $\mathrm{ma}$ & $\mathrm{mf}$ & $\mathrm{w}$ \\
\hline Cryptocarenus heveae & nd & $\mathrm{r}$ & pf & $\mathrm{w}$ & nd & c & $\mathrm{f}$ & $\mathrm{z}$ & nd & $\mathrm{d}$ & $\mathrm{pf}$ & $\mathrm{y}$ \\
\hline Cryptocarenus seriatus & nd & d & $\mathrm{pf}$ & $\mathrm{w}$ & nd & c & $\mathrm{f}$ & $\mathrm{y}$ & nd & c & $\mathrm{f}$ & $\mathrm{w}$ \\
\hline Cryptocarenus sp. & nd & $\mathrm{r}$ & $\mathrm{pf}$ & $\mathrm{z}$ & nd & d & $\mathrm{pf}$ & $\mathrm{z}$ & nd & $\mathrm{r}$ & $\mathrm{pf}$ & $\mathrm{z}$ \\
\hline Hypothenemus bolivianus & nd & $\mathrm{r}$ & $\mathrm{pf}$ & $\mathrm{y}$ & nd & $\mathrm{d}$ & $\mathrm{pf}$ & $\mathrm{z}$ & nd & $\mathrm{r}$ & $\mathrm{pf}$ & $\mathrm{y}$ \\
\hline Hypothenemus eruditus & $\mathrm{d}$ & c & $\mathrm{f}$ & $\mathrm{w}$ & nd & c & $\mathrm{f}$ & $\mathrm{w}$ & $\mathrm{d}$ & c & $\mathrm{f}$ & $\mathrm{w}$ \\
\hline Hypothenemus obscurus & nd & $\mathrm{r}$ & $\mathrm{pf}$ & $\mathrm{w}$ & nd & c & $\mathrm{f}$ & $\mathrm{w}$ & nd & $\mathrm{d}$ & $\mathrm{pf}$ & $\mathrm{w}$ \\
\hline Premnobius cavipennis & nd & $d$ & $\mathrm{pf}$ & $\mathrm{w}$ & $\mathrm{d}$ & $\mathrm{c}$ & $\mathrm{f}$ & $\mathrm{w}$ & nd & $\mathrm{c}$ & $\mathrm{f}$ & $\mathrm{w}$ \\
\hline Sampsonius dampfi & nd & $\mathrm{d}$ & $\mathrm{pf}$ & $\mathrm{w}$ & nd & c & $\mathrm{f}$ & $\mathrm{w}$ & nd & $\mathrm{d}$ & $\mathrm{pf}$ & $\mathrm{w}$ \\
\hline Xyleborus affinis & $\mathrm{d}$ & $\mathrm{ma}$ & $\mathrm{mf}$ & $\mathrm{w}$ & sd & sa & sf & $\mathrm{w}$ & $\mathrm{d}$ & $\mathrm{ma}$ & $\mathrm{mf}$ & $\mathrm{w}$ \\
\hline Xyleborus compactus & nd & $\mathrm{r}$ & pf & $\mathrm{w}$ & nd & $\mathrm{d}$ & $\mathrm{pf}$ & $\mathrm{z}$ & nd & $\mathrm{r}$ & $\mathrm{pf}$ & $\mathrm{y}$ \\
\hline Xyleborus ferrugineus & nd & c & $\mathrm{f}$ & $\mathrm{w}$ & $\mathrm{d}$ & $\mathrm{ma}$ & $\mathrm{mf}$ & $\mathrm{w}$ & $\mathrm{d}$ & $\mathrm{ma}$ & $\mathrm{mf}$ & $\mathrm{w}$ \\
\hline Xyleborus retusus & d & $\mathrm{ma}$ & $\mathrm{mf}$ & $\mathrm{w}$ & nd & c & $f$ & $\mathrm{z}$ & d & $\mathrm{a}$ & $\mathrm{mf}$ & $\mathrm{y}$ \\
\hline Xyleborus spinosulus & nd & $\mathrm{r}$ & $\mathrm{pf}$ & $\mathrm{z}$ & - & - & - & - & nd & $\mathrm{r}$ & $\mathrm{pf}$ & z \\
\hline Xyleborus spinulosus & nd & $\mathrm{d}$ & $\mathrm{pf}$ & $\mathrm{w}$ & - & - & - & - & nd & $\mathrm{d}$ & $\mathrm{pf}$ & $\mathrm{y}$ \\
\hline Tricolus sp. & - & - & - & - & nd & $\mathrm{d}$ & pf & $\mathrm{z}$ & nd & $\mathrm{r}$ & pf & $\mathrm{z}$ \\
\hline
\end{tabular}

Nota:D:Dominância - (sd) super dominante;(d) dominante; (nd) não dominante.A:Abundância - (sa) super abundante; (ma) muito abundante; (a) abundante; (c) comum; (d) dispersa; $(r)$ rara. F: Frequência - (sf) super frequente; (mf) muito frequente; (f) frequente; ( $p f)$ pouco frequente. C: Constância - (w) constante; $(y)$ acessória; $(z)$ acidental. 
predominância das espécies do gênero Chryptocarenus nos períodos de seca e de chuva na região.

\section{Diversidade}

No período de seca foram registrados os maiores valores de equitabilidade e diversidade (Shannon-Wiener), indicando que o ambiente fornece, neste período, condições favoráveis para uma distribuição mais homogênea das espécies. No período de chuva houve uma maior riqueza de espécies (Margalef), porém com uma população bastante heterogênea quanto à utilização dos recursos ambientais disponíveis neste ambiente (Tabela 4).

Diferindo dos resultados obtidos neste trabalho, Flechtmann e Ottati (1996) registraram em vegetação de cerrado, um maior índice de diversidade no período de chuva, quando comparado com os observados no período de seca e na análise anual.

\section{Flutuação Populacional}

As espécies $C$. diadematus e $X$. affinis ocorreram com picos populacionais em agosto. A espécie $X$. affinis também apresentou uma elevação na quantidade de indivíduos coletados nos meses de outubro (final do período seco), novembro e dezembro, meses de chuva na região, indicando que esta espécie inicia seu voo de dispersão e colonização de novos hospedeiros no final do período seco (Figura 1).

Dorvale Peres-Filho(2001) registraram pico populacional de C. diadematus em agosto, confirmando que esta espécie encontra, nos meses de seca, condições favoráveis para sua sobrevivência e desenvolvimento devido à abundância de hospedeiros neste ambiente. Comportamento semelhante foi observado por Diaz (1996) para X. affinis em mata nativa, no município de Tebicuary, no Paraguai, em que picos populacionais ocorreram em março, considerado final do período de chuva, em maio e agosto, meses do período de seca naquela região.

A população de B. uncinata apresentou um pico populacional em outubro, considerado final do período seco na região (Figura 1). Resultado semelhante ao observado por Flechtman et. al. (1997) em vegetação de cerrado, no município de Agudos, SP., onde B. uncinata ocorreu com picos populacionais em outubro e novembro, início do período de chuvas na região.

Xyleborus ferrugineus ocorreu com uma baixa densidade populacional, evidenciando que esta espécie apresenta normalmente populações com poucos indivíduos na área de estudo, independentemente do período analisado (Figura 1).

Tabela 4. Índices de equitabilidade, riqueza de Margalef e diversidade de Shannon-Wiener em um fragmento de cerrado no município de Cuiabá, estado de Mato Grosso, de abril/2008 a março/2009

\begin{tabular}{ccccccccc}
\hline \multicolumn{3}{c}{ Equitabilidade $(\mathrm{E})$} & \multicolumn{3}{c}{ Margalef $(\boldsymbol{\alpha})$} & \multicolumn{3}{c}{ Shannon-Wiener $(\mathrm{H})$} \\
\hline Seca & Chuva & Anual & Seca & Chuva & Anual & Seca & Chuva & Anual \\
\hline 0,7694 & 0,5493 & 0,7260 & 3,2932 & 3,3593 & 3,5434 & 2,3782 & 1,6456 & 2,3369 \\
\hline Nota: $E=\{0 ;$; $\}$.Variância & $H_{\text {SECA }}=0,0015$; Variância & $H_{\text {CHuva }}=0,0074$; Variância & $H_{\text {ANuAL }}=0,0013$. & &
\end{tabular}



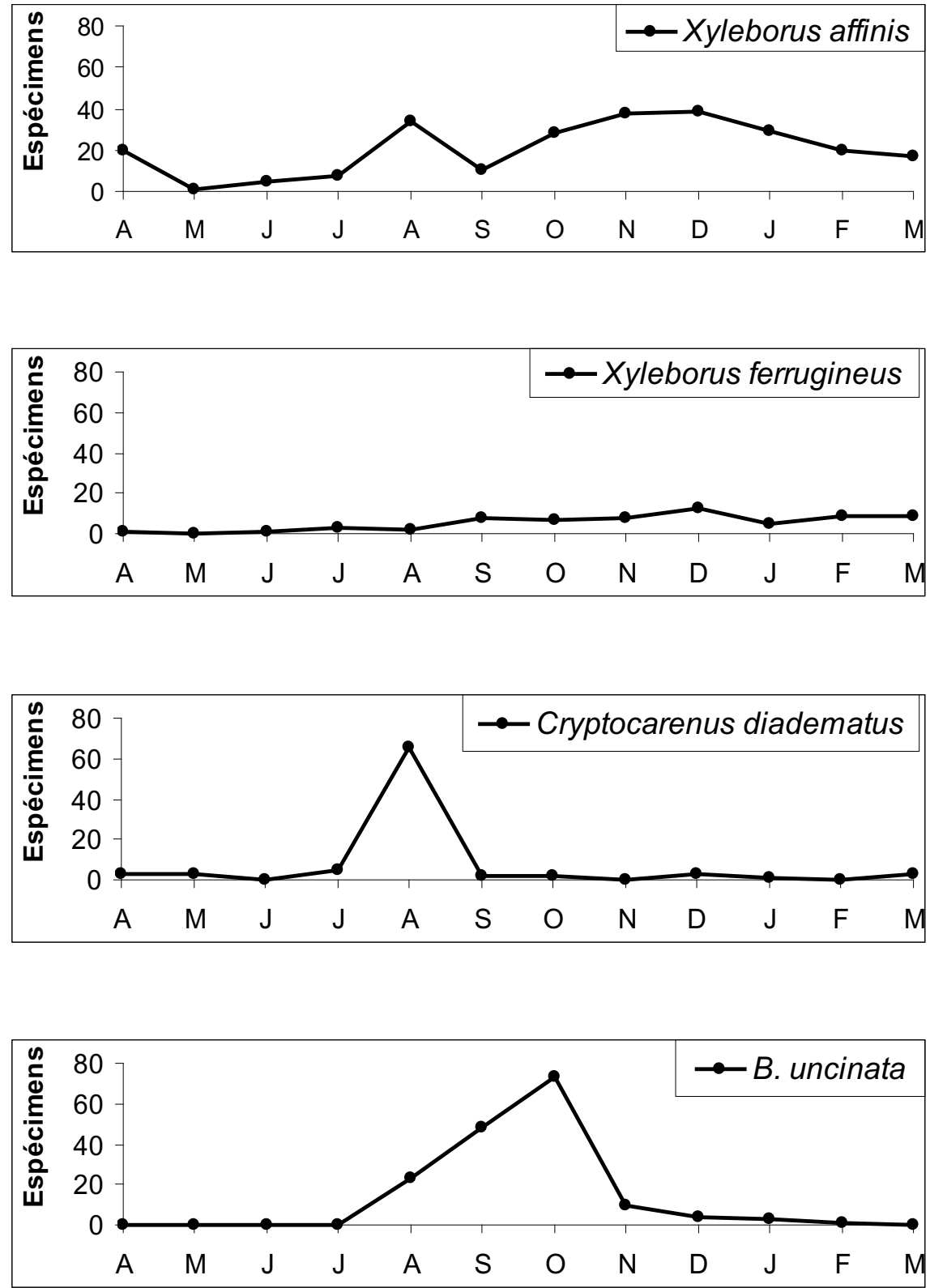

Figura I. Flutuação populacional de coleópteros em um fragmento de cerrado no município de Cuiabá, estado de Mato Grosso, de abril/2008 a março/2009 


\section{Correlação com Fatores Meteorológicos}

Dentre as espécies estudadas, apenas $C$. diadematus correlacionou-se significativamente com um dos fatores meteorológicos. Para esta espécie obteve-se correlação negativa ao nível de $72 \%$ com a umidade relativa do ar. Este resultado corrobora os resultados de Dorval e PeresFilho (2001), em vegetação de cerrado, que encontraram para esta espécie, correlações negativa com umidade relativa, precipitação pluvial, temperatura média e mínima, evidenciando a preferência de $C$. diadematus por ocorrência nos meses do período seco.

\section{Testes de Médias}

A análise de variância para as espécies identificadas no fragmento de cerrado revelou diferenças estatísticas significativas ao nível de $1 \%$ de probabilidade para todas as fontes de variação analisadas (Tabela 5).

Observa-se, na tabela 6, que ocorreram seis agrupamentos de médias na avaliação anual, sendo $X$. affinis seguido de $B$. uncinata diferenciaram-se das demais espécies, ocorrendo isoladamente e com as maiores médias populacionais. Nos períodos de chuva e seca, $X$. affinis e B. uncinata, respectivamente, ocorreram com as maiores médias de indivíduos coletados.

Para as espécies B. uncinata, Xyloprista sp., P. linearis, C. diadematus, C. seriatus, $H$. eruditus, $X$. Retusus e $X$. affinis obtiveramse médias diferentes estatísticamente entre períodos de seca e de chuva. Estes resultados indicam a forte influência dos períodos meteorólogicos de seca e de chuva sobre estas espécies na região.

Diferentemente deste estudo, em que $X$. affinis aparece como a espécie de maior média neste ambiente, Dorval e Peres-Filho (2001) observaram para a mesma região, que $C$. herveae, C. seriatus e $H$. obscurus formaram um grupo com as maiores médias de indivíduos coletados, concluindo que existe uma alternância de posições destas espécies ao longo do ano e que são fortemente influenciadas pelas condições ambientais e pelo grau de adaptação destas espécies às condições locais.

Com base nos resultados obtidos, concluiu-se que, independente dos meses de seca e de chuva, a família Scolytidae foi a mais representativa em quantidade de espécies e de indivíduos coletados, indicando estar mais adptada às condições ambientais da região, quando comparada com as demais espécies das famílias estudadas.

Tabela 5. Análise de variância dos coleópteros coletados em um fragmento de cerrado no município de Cuiabá, estado de Mato Grosso, de abril/2008 a março/2009

\begin{tabular}{llllll}
\hline Fontes de Variação & G.L. & S.Q. & Q.M. & Fc. & Signif. \\
\hline Períodos & 1 & 3,4583 & 3,4583 & $111,60^{* *}$ & 0,00004 \\
Espécies & 24 & 30,6937 & 1,2789 & $41,27^{* *}$ & 0,00000 \\
Períodos x Espécies & 24 & 9,2821 & 0,3868 & $12,48^{* *}$ & 0,00000 \\
Resíduo & 250 & 7,7473 & 0,0310 & & \\
\hline Total & 299 & 51,1815 & & & \\
\hline
\end{tabular}

Transf.: $\log (\mathrm{x}+1)$; C.V. $(\%)=53,959$

Nota: ** Significativo ao nível de $1 \%$ de probabilidade pelo teste $F$. 
Tabela 6. Teste de média entre as espécies de coleópteros coletados em um fragmento de cerrado no município de Cuiabá, estado de Mato Grosso, de abril/2008 a março/2009

\begin{tabular}{|c|c|c|c|c|c|c|}
\hline \multirow{2}{*}{ Espécies } & \multicolumn{6}{|c|}{ Médias } \\
\hline & \multicolumn{2}{|c|}{ Seca } & \multicolumn{2}{|c|}{ Chuva } & \multicolumn{2}{|c|}{ Anual } \\
\hline Bostrychopsis laminifer & - & - & 0,2300 & $\mathrm{D}$ & 0,1150 & $\mathrm{~F}$ \\
\hline Bostrychopsis uncinata & 1,3538 & $\mathrm{Aa}$ & 0,5584 & $\mathrm{Cb}$ & 0,9561 & $\mathrm{~B}$ \\
\hline Micrapate sp. & 0,8675 & $\mathrm{C}$ & - & - & 0,4338 & $\mathrm{E}$ \\
\hline Xyloperthela picea & 0,6881 & $\mathrm{C}$ & - & - & 0,3440 & $\mathrm{E}$ \\
\hline Xyloprista sp. & 0,3597 & $\mathrm{Da}$ & 0,1003 & $\mathrm{~Eb}$ & 0,2300 & $\mathrm{E}$ \\
\hline Platypus linearis & 0,7999 & $\mathrm{Ca}$ & 0,1799 & $\mathrm{~Eb}$ & 0,4899 & $\mathrm{D}$ \\
\hline Platypus sp. & 0,0502 & $\mathrm{Ea}$ & 0,0502 & $\mathrm{Ea}$ & 0,0502 & $\mathrm{~F}$ \\
\hline Coccotrypes sp. & - & - & 0,0502 & $\mathrm{E}$ & 0,0251 & $\mathrm{~F}$ \\
\hline Cortbylus convexicauda & 0,0502 & $\mathrm{E}$ & - & - & 0,0251 & $\mathrm{~F}$ \\
\hline Cryptocarenus diadematus & 1,1111 & $\mathrm{Ba}$ & 0,4099 & $\mathrm{Cb}$ & 0,7605 & $\mathrm{C}$ \\
\hline Cryptocarenus heveae & 0,1297 & $\mathrm{Ea}$ & 0,1799 & $\mathrm{Ea}$ & 0,1548 & $\mathrm{~F}$ \\
\hline Cryptocarenus seriatus & 0,4052 & $\mathrm{Da}$ & 0,1505 & $\mathrm{~Eb}$ & 0,2779 & $\mathrm{E}$ \\
\hline Cryptocarenus sp. & 0,0502 & $\mathrm{Ea}$ & 0,0502 & $\mathrm{Ea}$ & 0,0502 & $\mathrm{~F}$ \\
\hline Hypothenemus bolivianus & 0,1003 & $\mathrm{Ea}$ & 0,0502 & $\mathrm{Ea}$ & 0,0753 & $\mathrm{~F}$ \\
\hline Hypothenemus eruditus & 0,7787 & $\mathrm{Ca}$ & 0,2802 & $\mathrm{Db}$ & 0,5295 & $\mathrm{D}$ \\
\hline Hypothenemus obscurus & 0,2092 & $\mathrm{Da}$ & 0,1505 & $\mathrm{Ea}$ & 0,1799 & $\mathrm{~F}$ \\
\hline Premnobius cavipennis & 0,2802 & $\mathrm{Da}$ & 0,3806 & $\mathrm{Ca}$ & 0,3304 & $\mathrm{E}$ \\
\hline Sampsonius dampfi & 0,2802 & $\mathrm{Da}$ & 0,2300 & $\mathrm{Da}$ & 0,2551 & $\mathrm{E}$ \\
\hline Xyleborus affinis & 1,1617 & $\mathrm{Bb}$ & 1,4124 & $\mathrm{Aa}$ & 1,2870 & A \\
\hline Xyleborus compactus & 0,1297 & $\mathrm{Ea}$ & 0,0502 & $\mathrm{Ea}$ & 0,0899 & $\mathrm{~F}$ \\
\hline Xyleborus ferrugineus & 0,6221 & $\mathrm{Ca}$ & 0,8079 & $\mathrm{Ba}$ & 0,7150 & $\mathrm{C}$ \\
\hline Xyleborus retusus & 1,0026 & $\mathrm{Ba}$ & 0,1003 & $\mathrm{~Eb}$ & 0,5515 & $\mathrm{D}$ \\
\hline Xyleborus spinosulus & 0,0502 & $\mathrm{E}$ & - & - & 0,0251 & $\mathrm{~F}$ \\
\hline Xyleborus spinulosus & 0,3597 & $\mathrm{D}$ & - & - & 0,1799 & $\mathrm{~F}$ \\
\hline Tricolus sp. & - & - & 0,0502 & $\mathrm{E}$ & 0,0251 & $\mathrm{~F}$ \\
\hline Médias de Períodos & 0,4336 & $\mathrm{a}$ & 0,2189 & $\mathrm{~b}$ & & \\
\hline
\end{tabular}

Nota:Médias acompanhadas de mesma letra maiúscula em coluna, ou de mesma letra minúscula em linha, não diferem entre si ao nível de $5 \%$ de probabilidade pelo teste de Scott-Knott.

\section{Referências}

BERTI-FILHO, E. Impacto de Coleoptera Cerambycidae em florestas de Eucalyptus no Brasil. Scientia Forestalis, Piracicaba, v. 52, p. 51-54 p. 1997.

CAMPOS, H. Estatística experimental não-paramétrica. Piracicaba: EDUSP,1976. 322 p.

CARRANO-MOREIRA, A.F.; PEDROSA-MACEDO, J.H. Levantamento e análise faunística da família Scolytidae (Coleoptera) em comunidades florestais no estado do Paraná. Anais da Sociedade Entomológica do Brasil, Londrina, v. 23, n. 1, p.115-26, 1994. 
CONCEIÇÃO, P. N. (Coordenador). Manejo de bacia hidrogáfica do rio Coxipó-Açú para concervação de seus recursos hídricos. Brasília: ABEAS/ MMA/ SRH/UFMT, 127p. 1997.

COSTA NETO, P. L. O. Estatística. São Paulo: Edgard Blücher, 1977. 168 p.

DIAZ, E. A. B.Análise faunística de Scolytidae, Platypodidae e Bostrichidae (Coleoptera) em comunidades florestais no Departemento de Guairá, Paraguai. 1996. 53f. Dissertação (Mestrado em Ciências Biológicas) - Universidade Federal do Paraná, UFPR, Curitiba, 1996.

DORVAL, A. Levantamento populacional de coleópteros com armadilhas etanólicas em plantios de eucaliptos e em uma área com vegetação de cerrado no município de Cuiabá, Estado de Mato Grosso. 2002. 143f. Tese (Doutorado em Engenharia Florestal) - Universidade Federal do Paraná, Curitiba, 2002.

DORVAL, A.; PERES-FILHO, O. Levantamento e flutuação populacional de coleópteros em vegetação do cerrado da baixada Cuiabana, MT. Ciência Florestal. Santa Maria-RS, v. 11, n. 2, p. 171-182. 2001.

FLECHTMANN, C. A. H.; OTTATI, A. L. T. Scolytidae em area de mata nativa em Selvíria, MS, Brasil. Anais da Sociedade Entomológica do Brasil. Londrina, v. 25, n. 2, p. 365-368. 1996.

FLETCHMANN, C. A. H.; GASPARETO, C. L.; TEIXEIRA, E. P. Bostrichidae in a stand of cerrado in Agudos, São Paulo State. Revista do Instituto Florestal, São Paulo, v. 8, n.1, p. 45-50. 1997.

FURNISS, R. L.; CAROLIN, V.W. Western forest insects. Washington, USDA Forest Service, 1977, 654p.

HILL, M. G.; NANG'AYO, F. L. O.; WRIGHT, D. J. Biological control of the larger grain borer Prostephanus truncatus (Coleoptera: Bostrichidae) in Kenya using a predatory beetle Teretrius nigrescens (Coleoptera: Histeridae). Bulletin of Entomological Research. v. 93, n.4, [S.I.], p. 299-306. 2003.

MARINONI, R. C.; GANHO, N. G.; MONNÉ, M. L.; MERMUDES, J. R. M. Hábitos alimentares em Coleoptera (Insecta). Ribeirão Preto, Holos, 2001. 64p.

MARQUES, E. N. Scolytidae e Platypodidae em Pinus taeda. 1984. 65f. Dissertação (mestrado em Engenharia Florestal) - Universidade Federal do Paraná, UFPR, Curitiba, 1984. UFPR. Curitiba.

MATOSKI, S. L. S. Comportamento de Dinoderus minutus Fabricius (1775) (Coleoptera: Bostrichidae) em lâminas torneadas de madeira. 2005, 94f. Dissertação de Mestrado em Engenharia Florestal. - Universidade Federal do Paraná, UFPR, Curitiba, 2005. 
MORAES, R. C. B. ; SILVEIRA NETO, S. Software para análise faunística. In: SIMPÓSIO DE CONTROLE BIOLÓGICO, 8., São Paulo, Resumos, Piracicaba-SP. ESALQ.p. 95. 2003.

SILVA, A. G. A.; GONÇALVES, C. R.; GALVÃO, D.M.; GONÇALVES. A. J. L.; GOMES J.; SILVA, M.N. SIMONI, L. Quarto catálago dos insetos que vivem nas plantas do Brasil e seus parasitos e predadores. Parte II - 10 Tomo. Departamento de Defesa e Inspeção Agropecuária - Ministério da Agricultura. Rio de Janeiro, 1968. 622p.

SIMEONE, J. B.. Insects and wood. New York: Syracuse, 1965. 178p. 